\title{
Green functions on product networks
}

\author{
C. Araúz ${ }^{\mathrm{a}}$, Á. Carmona ${ }^{\mathrm{b}}$, A.M. Encinas ${ }^{\text {b,* }}$, M. Mitjana ${ }^{\mathrm{b}}$ \\ a Institut Cerdà, Barcelona, Spain \\ b Departament de Matemàtiques, Universitat Politècnica de Catalunya, Barcelona, Spain
}

\section{A R T I C L E I N F O}

\section{Article history:}

Received 29 September 2017

Received in revised form 27 September 2018

Accepted 1 October 2018

Available online $\mathrm{xxxx}$

\section{Keywords:}

Discrete potential theory

Schrödinger operators

Green functions

Product networks

Spectra

Separation of variables

\begin{abstract}
A B S T R A C T
We aim here at determining the Green function for general Schrödinger operators on product networks. The first step consists in expressing Schrödinger operators on a product network as sum of appropriate Schrödinger operators on each factor network. Hence, we apply the philosophy of the separation of variables method in PDE, to express the Green function for the Schrödinger operator on a product network using Green functions on one of the factors and the eigenvalues and eigenfunctions of some Schrödinger operator on the other factor network. We emphasize that our method only needs the knowledge of eigenvalues and eigenfunctions of one of the factors, whereas other previous works need the spectral information of both factors. We apply our results to compute the Green function of $P_{m} \times S_{h}$, where $P_{m}$ is a Path with $m$ vertices and $S_{h}$ is a Star network with $h+1$ vertices.
\end{abstract}

(C) 2018 Elsevier B.V. All rights reserved.

\section{Introduction}

Green's functions on a network are closely related with self-adjoint boundary value problems for Schrödinger operators. Although there exists a very interesting variety of such a boundary value problems, see for instance [3], we restrict ourselves here to analyze either the Dirichlet Problem or the Poisson equation. As mentioned in a recent paper by A. Gilbert et alt.: "The idea of discrete Green's functions has, implicitly or explicitly, a long history arising in many important problems and fields such as the study of inverses of tri-diagonal matrices, potential theory, the study of Schrödinger operators on graphs, and the graph-theoretic analog of Poisson's equation. Additionally, Green's function methods have yielded interesting results in many areas including the properties of random walks, chip-firing games, analysis of online communities, machine learning algorithms and load balancing in networks.", see [15] and references therein.

In spite of its importance, only few explicit expressions for Green's functions associated with Schrödinger operators on very structured networks, are known. The most common technique to get these expressions consists in using the spectral decomposition property. So, in general, determining Green's functions is a very difficult task. Another strategy to compute these elements is to split the network into small and structured pieces and then to express the eigenvalues and eigenfunctions in terms of those corresponding to each piece. Since composite networks as join, corona or cluster have been studied in a very general setting, see [1,5] and also [16] for graphs, we analyze here the case of cartesian product of networks. As we show, our treatment is the discrete version of the Fourier Method, also called, Separation of Variables Method. We first prove that when we consider weights that are tensor product of weights, then the corresponding Schrödinger operators are expressed in separated variables and hence the Fourier Method fits accurately.

\footnotetext{
* Corresponding author.

E-mail addresses: carauz@icerda.org (C. Araúz), angeles.carmona@upc.edu (Á. Carmona), andres.marcos.encinas@upc.edu (A.M. Encinas), margarida.mitjana@upc.edu (M. Mitjana).
} 


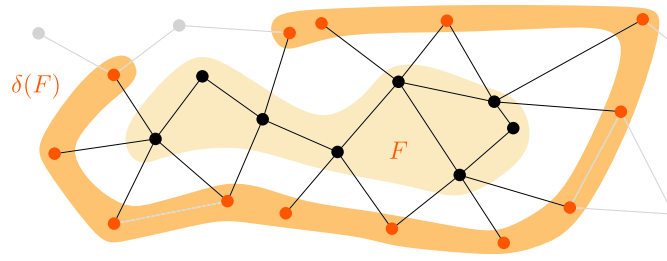

Fig. 1. A vertex set $F(\bullet)$ and its boundary $\delta(F)(\bullet)$.

This class of problems have been also studied by F. Chung, R. Ellis and S.T. Yau, see [11,13,14], considering the normalized Laplacian. However, since in general the normalized Laplacian of a product network cannot be described in separated variables involving the normalized Laplacian of the factor networks, in the above referred works the authors must consider only cartesian product of regular networks, that is also a regular network. We remark that in this case, the problem is reduced to the analysis of the combinatorial Laplacian, since for regular networks the normalized Laplacian is a multiple of the combinatorial one. We treat here with Schrödinger operators on the product network without any assumption on the regularity of each factor network, but under the hypothesis that the potential is related with tensor product of weights. We emphasize that the application of the separation of variable method only requires the knowledge of eigenvalues and eigenfunctions of one of the factors. So, applying our results we can get explicit expressions for Green's functions in a wide range of product networks.

A finite network $\Gamma=(V, c)$, consists of a finite set $V$, called vertex set and a symmetric function $c: V \times V \longrightarrow[0,+\infty)$, called conductance, satisfying that $c(x, x)=0$ for any $x \in V$. Two vertices $x, y \in V$ are adjacent iff $c(x, y)>0$.

We always assume that $\Gamma$ is connected; that is, that for any pair of different vertices $x, y \in V$, there exist $m \in \mathbb{N}^{*}$ and $x_{0}, \ldots, x_{m} \in V$ such that $x=x_{0}, y=x_{m}$ and $\prod_{j=0}^{m-1} c\left(x_{j}, x_{j+1}\right)>0$.

In what follows $\mathcal{C}(V)=\mathcal{C}(V ; \mathbb{R})$ and $\mathcal{C}(V ; \mathbb{C})$ stand respectively for the spaces of real and complex functions defined on the vertex set $V$. Given $v \in \mathcal{C}(V ; \mathbb{C}), \bar{v}$ denotes its conjugate and then, $\langle u, v\rangle=\sum_{x \in V} u(x) \bar{v}(x)$ determines an inner product on $\mathcal{C}(V ; \mathbb{C})$, whose associated norm is denoted by $\|\cdot\|$. Therefore, $\|u\|=\left(\sum_{x \in V}|u(x)|^{2}\right)^{\frac{1}{2}}$ for any $u \in \mathcal{C}(V ; \mathbb{C})$. Clearly, this inner product induces the standard one on $\mathcal{C}(V)$. Given $u \in \mathcal{C}(V, \mathbb{C}), u^{\perp}$ denotes the subspace of $\mathcal{C}(V, \mathbb{C})$ orthogonal to $u$.

A real-valued function $\omega \in \mathcal{C}(V)$ is called weight if $\omega(x)>0$ for any $x \in V$ and in addition $\|\omega\|=1$. The sets of weights on $V$ is denoted by $\Omega(V)$ or simply by $\Omega$ when it does not lead to confusion. Clearly the weight $v$ defined as $v(x)=|V|^{-\frac{1}{2}}$, $x \in V$, is the unique constant weight on $V$.

For any $x \in V, \varepsilon_{x}$ is the Dirac function at $x$. Clearly $\varepsilon_{x} \in \mathcal{C}(V)$ for any $x \in V$. Moreover, $\kappa$ denotes the (generalized) degree of $\Gamma$; that is, the function defined as $\kappa(x)=\sum_{y \in V} c(x, y)$, for any $x \in V$. The network is called regular when its degree is a constant function. The volume of the network $\Gamma$ is $v=\sum_{x \in V} \kappa(x)$. Since $\Gamma$ is connected $v^{-\frac{1}{2}} \sqrt{\kappa}$ is a weight, called the volume weight.

Given $F \subset V$ a nonempty subset, $F^{c}$ denotes its complementary and $\mathcal{C}(F)$ and $\mathcal{C}(F ; \mathbb{C})$ are the subspaces of real and complex functions vanishing on $F^{c}$. It is clear that $\mathcal{C}(F)$ and $\mathcal{C}(F ; \mathbb{C})$ can be identified respectively with the space of real or complex functions defined on $F$. Moreover, the set

$$
\delta(F)=\left\{z \in F^{c}: c(z, y)>0 \text { for some } y \in F\right\}
$$

is called the boundary of $F$ and then, $\bar{F}=F \cup \delta(F)$ is the closure $F$, see Fig. 1. Clearly, $\delta(F)=\emptyset$, or equivalently $F=\bar{F}$, iff $F=V$.

Analogously, given $F \subset V$, each function $K: F \times F \longrightarrow \mathbb{R}$ can be identified as a function on $V \times V$ vanishing outside of $F \times F$. The above function is called symmetric if it satisfies that $K(x, y)=K(y, x)$ for any $x, y \in F$. Clearly if we consider $K$ extended by 0 on $(F \times F)^{c}$, then $K$ is symmetric on $V \times V$ iff it is symmetric on $F \times F$.

The combinatorial Laplacian of $\Gamma$, or simply the Laplacian of $\Gamma$, is the linear operator $\mathcal{L}: \mathcal{C}(V ; \mathbb{C}) \longrightarrow \mathcal{C}(V ; \mathbb{C})$ that assigns to any $u \in \mathcal{C}(V ; \mathbb{C})$ the function $\mathcal{L}(u)$ defined as

$$
\mathcal{L}(u)(x)=\sum_{y \in V} c(x, y)(u(x)-u(y)), \quad x \in V .
$$

More generally, given $q \in \mathcal{C}(V ; \mathbb{C})$, the Schrödinger operator with potential $q$, see [7], is $\mathcal{L}_{q}: \mathcal{C}(V ; \mathbb{C}) \longrightarrow \mathcal{C}(V ; \mathbb{C})$ defined as $\mathcal{L}_{q}(u)=\mathcal{L}(u)+q u$ for any $u \in \mathcal{C}(V ; \mathbb{C})$. The Schrödinger operator whose potential is the conjugate of $q$; that is, $\mathcal{L}_{\bar{q}}$, is called the adjoint of $\mathcal{L}_{q}$ since it satisfies that $\left\langle\mathcal{L}_{q}(u), v\right\rangle=\left\langle u, \mathcal{L}_{\bar{q}}(v)\right\rangle$ for any $u, v \in \mathcal{C}(V ; \mathbb{C})$.

For a given nonempty subset $F \subset V$ and a given potential $q \in \mathcal{C}(V ; \mathbb{C})$ we consider the following Boundary Value Problem: Given $f \in \mathcal{C}(F ; \mathbb{C})$ and $g \in \mathcal{C}(\delta(F) ; \mathbb{C})$, find $u \in \mathcal{C}(F ; \mathbb{C})$ such that

$$
\mathcal{L}_{q}(u)=f \text { on } F, \quad u=g \text { on } \delta(F) .
$$

When $F \neq V$, this problem is known as Dirichlet Problem on $F$, whereas when $F=V$ it is called Poisson equation on $V$. In this last case the data $g$ has no sense, since then $\delta(F)=\emptyset$. 
When $F \neq V$, each Dirichlet problem on $F$ is equivalent to a semihomogeneous Dirichlet problem. Specifically, $u \in \mathcal{C}(\bar{F} ; \mathbb{C})$ is a solution of Problem (1) iff $v=u-g$ is a solution of the Dirichlet problem

$$
\mathcal{L}_{q}(u)=f-\mathcal{L}(g) \text { on } F, \quad u=0 \text { on } \delta(F) .
$$

Therefore, to analyze the existence and uniqueness of solution of the boundary value problem for any $f \in \mathcal{C}(F ; \mathbb{C})$ is equivalent to analyze the same topics for the following problem:

$$
\text { Given } f \in \mathcal{C}(F ; \mathbb{C}) \text {, find } u \in \mathcal{C}(F ; \mathbb{C}) \text { such that } \mathcal{L}_{q}(u)=f \text { on } F \text {. }
$$

This formulation encompasses both, Dirichlet problems and Poisson equations; the last ones appear when $F=V$.

Notice that Schrödinger operators with real-valued potential; that is, $\mathcal{L}_{q}$ with $q \in \mathcal{C}(V)$ are also endomorphisms on $\mathcal{C}(V)$ and moreover, they are self-adjoint; since $\left\langle\mathcal{L}_{q}(u), v\right\rangle=\left\langle u, \mathcal{L}_{q}(v)\right\rangle$ for any $u, v \in \mathcal{C}(V ; \mathbb{C})$. In particular, $\sum_{x \in F} \mathcal{L}_{q}(u)(x) v(x)=$ $\sum_{x \in F} \mathcal{L}_{q}(v)(x) u(x)$ for any $u, v \in \mathcal{C}(F ; \mathbb{C})$; that means that Problem $(3)$ is self-adjoint on $\mathcal{C}(F ; \mathbb{C})$ when the potential is realvalued.

This work is mainly concerned with Schrödinger operators with real-valued potentials and for this reason we usually consider only real-valued functions; that is, the space $\mathcal{C}(V)$. Moreover, in this case we also consider the Energy for the potential $q$ that is the quadratic form $\mathcal{E}_{q}: \mathcal{C}(V) \longrightarrow \mathbb{R}$ that assigns to any $u \in \mathcal{C}(V)$ the value

$$
\mathcal{E}_{q}(u)=\left\langle\mathcal{L}_{q}(u), u\right\rangle=\frac{1}{2} \sum_{x, y \in V} c(x, y)(u(x)-u(y))^{2}+\sum_{x \in V} q(x) u(x)^{2} .
$$

\section{Real-valued potentials and Doob transforms}

For any weight $\omega \in \Omega$, we call the function $q_{\omega}=-\omega^{-1} \mathcal{L}(\omega)$ Doob potential associated with $\omega$. Therefore,

$$
q_{\omega}(x)=-\kappa(x)+\omega(x)^{-1} \sum_{y \in V} c(x, y) \omega(y)>-\kappa(x), \quad \text { for any } x \in V .
$$

Given two weights $\sigma, \omega \in \Omega$, then $q_{\sigma} \geq q_{\omega}$ iff $q_{\sigma}=q_{\omega}$ and this happens iff $\sigma=\omega$, see [4, Lemma 2.1]. In particular, $q_{\sigma}=0$ iff $\sigma$ is constant and hence, $q_{\sigma}$ takes positive and negative values when $\sigma \in \Omega$ is not constant. Notice that $\left\langle\sigma, q_{\sigma}\right\rangle=-\langle 1, \mathcal{L}(\sigma)\rangle=0$.

Although at first glance Doob transforms could seem a bit strange and Doob potentials a very specific kind of potentials, they play a main role among real-valued potentials. In fact, as a consequence of the Perron-Frobenius Theory, given a realvalued potential $q \in \mathcal{C}(V)$ there exist a unique unitary weight $\omega \in \Omega$ and a unique real value $\lambda \in \mathbb{R}$ such that $q=q_{\omega}+\lambda$, see [2]. The following result involving Doob potentials has been strongly used by the authors, see for instance [2,4].

Proposition 2.1 (Doob Transform). Let a real-valued potential $q$ and consider $\omega \in \Omega$ and $\lambda \in \mathbb{R}$ such that $q=q_{\omega}+\lambda$. Then, if $F \subset V$ is a non-empty subset, for any $u \in \mathcal{C}(F)$ we have that

$$
\begin{aligned}
\mathcal{L}_{q}(u)(x) & =\frac{1}{\omega(x)} \sum_{y \in \bar{F}} c(x, y) \omega(x) \omega(y)\left(\frac{u(x)}{\omega(x)}-\frac{u(y)}{\omega(y)}\right)+\lambda u(x), \quad x \in F \\
\mathcal{E}_{q}(u) & =\frac{1}{2} \sum_{x, y \in \bar{F}} c(x, y) \omega(x) \omega(y)\left(\frac{u(x)}{\omega(x)}-\frac{u(y)}{\omega(y)}\right)^{2}+\lambda\|u\|^{2} .
\end{aligned}
$$

As we will show, these expressions have interesting consequences in the treatment of the boundary value problems we have raised.

We first remark that the well-known normalized Laplacian introduced in 1996 by F. Chung and R. Langlands, see [9-11], is nothing else but a Schrödinger operator on an appropriate network. The normalized Laplacian for the network $\Gamma=(V, c)$ is the operator $\mathscr{L}: \mathcal{C}(V) \longrightarrow \mathcal{C}(V)$ that assigns to any $u \in \mathcal{C}(V)$ the function

$$
\mathscr{L}(u)(x)=\frac{1}{\sqrt{\kappa(x)}} \sum_{x, y \in V} c(x, y)\left(\frac{u(x)}{\sqrt{\kappa(x)}}-\frac{u(y)}{\sqrt{\kappa(y)}}\right), \quad x \in V .
$$

Therefore, if $\mathcal{L}$ is the combinatorial Laplacian of $\Gamma$ and $\mathcal{T}: \mathcal{C}(V) \longrightarrow \mathcal{C}(V)$ is given by $\mathcal{T}(u)=\sqrt{\kappa} u$, we get that $\mathscr{L}=$ $\mathcal{T}^{-1} \circ \mathcal{L} \circ \mathcal{T}^{-1}$.

Moreover, we consider now the network $\widehat{\Gamma}=(V, \hat{c})$, where $\hat{c}(x, y)=\frac{c(x, y)}{\sqrt{\kappa(x) \kappa(y)}}$ for any $x, y \in V$ and $\hat{\mathcal{L}}$ its combinatorial Laplacian. Notice that any pair of vertices $x, y \in V$ are adjacent in $\Gamma$ iff they are adjacent in $\widehat{\Gamma}$, so the graphs subjacent to $\Gamma$ and to $\widehat{\Gamma}$ are the same. Choosing the volume weight of $\Gamma, \omega=\mathrm{v}^{-\frac{1}{2}} \sqrt{\kappa}$, from the expression for $\hat{\mathcal{L}}_{q_{\omega}}$ obtained after the Doob Transform associated with $\omega$, for any $u \in \mathcal{C}(V)$ and any $x \in V$, we have

$$
\hat{\mathcal{L}}_{q_{\omega}}(u)(x)=\frac{\sqrt{v}}{\sqrt{\kappa(x)}} \sum_{y \in V} \frac{1}{v} \hat{c}(x, y) \sqrt{\kappa(x)} \sqrt{\kappa(y)}\left(\frac{\sqrt{v} u(x)}{\sqrt{\kappa(x)}}-\frac{\sqrt{v} u(y)}{\sqrt{\kappa(y)}}\right)=\mathscr{L}(u)(x),
$$

and hence, the normalized Laplacian $\mathscr{L}$ on $\Gamma$ is equivalent to the Schrödinger operator $\hat{\mathcal{L}}_{q_{\omega}}$ on $\widehat{\Gamma}$. 
On the other hand, from the expression for the energy obtained after the Doob Transform, we have that

$$
\min _{\substack{u \in \mathcal{C}(F) \\\|u\|=1}}\left\{\mathcal{E}_{q}(u)\right\} \geq \lambda
$$

and the equality holds iff $F=V$. In this case, $\mathcal{E}_{q}$ attains its minimum at $u= \pm \omega$. Therefore, the Schrödinger operator $\mathcal{L}_{q}$ is positive semidefinite on $\mathcal{C}(V)$; that is, its energy is non-negative, iff $\lambda \geq 0$ and positive definite on $\mathcal{C}(V)$ iff $\lambda>0$. In addition, when $\lambda \geq 0$, the Schrödinger operator $\mathcal{L}_{q}$ is positive definite on $\mathcal{C}(F)$ for any proper subset $F$.

The variational characterization of the solutions for the boundary value problems (3) is described in the following result, see [4, Proposition 3.5] for its proof.

Proposition 2.2 (Dirichlet Principle). Let $F \subset V$ be a non empty subset, $\omega \in \Omega, \lambda \geq 0$ and the potential $q=q_{\omega}+\lambda$. Given $f \in \mathcal{C}(F)$ consider the quadratic functional $\mathcal{J}: \mathcal{C}(V) \longrightarrow \mathbb{R}$ given by

$$
\mathcal{J}(u)=\mathcal{E}_{q}(u)-2\langle f, u\rangle .
$$

Then $u \in \mathcal{C}(F)$ satisfies that $\mathcal{L}_{q}(u)=f$ on $F$ iff it minimizes $\mathcal{J}$ on $\mathcal{C}(F)$. Moreover $\mathcal{J}$ has a unique minimum except when $F=V$ and $\lambda=0$ simultaneously. In this case $\mathcal{J}$ has a minimum iff $f \in \omega^{\perp}$ and moreover, there exists a unique minimum belonging to $\omega^{\perp}$.

\section{Green functions, eigenvalues and eigenfunctions}

In this section we consider fixed the finite and connected network $\Gamma=(V, c)$, a weight $\omega \in \Omega$, a non-negative value $\lambda \geq 0$, the real-valued potential $q=q_{\omega}+\lambda$ and its corresponding Schrödinger operator $\mathcal{L}_{q}$. Under these hypotheses, for any proper subset $F \subset V$ and any $f \in \mathcal{C}(F)$, Dirichlet Problem (3) has a unique solution; that is, there exists a unique $u \in \mathcal{C}(F)$ such that $\mathcal{L}_{q}(u)=f$ on $F$. Moreover, when $\lambda>0$ for any $f \in \mathcal{C}(V)$, Poisson equation (3) has a unique solution; that is, there exists a unique $u \in \mathcal{C}(V)$ such that $\mathcal{L}_{q}(u)=f$ on $V$.

When either $F \subset V$ is a proper subset or $\lambda>0$, the Green function of $F$ for the potential $q$ is $G_{q}^{F}: F \times F \longrightarrow \mathbb{R}$ such that for any $y \in F, G_{q}^{F}(\cdot, y)$ is the unique solution of the Dirichlet Problem $\mathcal{L}_{q}(u)=\varepsilon_{y}$ on $F, u=0$ en $\delta(F)$, when $F$ is proper, or the Poisson equation $\mathcal{L}_{q}(u)=\varepsilon_{y}$ on $V$ when $F=V$ but $\lambda>0$.

The Green operator of $F$ for the potential $q$ is $\mathcal{G}_{q}^{F}: \mathcal{C}(F) \longrightarrow \mathcal{C}(F)$ defined for any $f \in \mathcal{C}(F)$ as $\mathcal{G}_{q}^{F}(f)(x)=\sum_{y \in F} G_{q}^{F}(x, y) f(y)$, $x \in F$. Then $\mathcal{G}_{q}^{F}$ is self-adjoint and for any $f \in \mathcal{C}(F)$, the function $u=\mathcal{G}_{q}^{F}(f) \in \mathcal{C}(F)$ satisfies that $\mathcal{L}_{q}(u)=f$ on $F$. Since $\mathcal{G}_{q}^{F}$ is a self-adjoint operator, then $G_{q}^{F}$ is a symmetric function and the Minimum Principle also implies that $0<\omega(y) G_{q}^{F}(x, y)<$ $\omega(x) G_{q}^{F}(y, y)$ for any $x, y \in F$, see for instance [4].

When $\lambda=0$, then $q=q_{\omega}$ and the Poisson equation $\mathcal{L}_{q}(u)=f$ on $V$ is solvable only if $f \in \omega^{\perp}$ and in this case, there exists a unique solution belonging to $\omega^{\perp}$. The Green function of $V$ for the potential $q$ is $G_{q}^{V}: V \times V \longrightarrow \mathbb{R}$ such that for any $y \in V$, $G_{q}^{V}(\cdot, y)$ is the unique solution of the Poisson equation $\mathcal{L}_{q}(u)=\varepsilon_{y}-\omega(y) \omega$ belonging to $\omega^{\perp}$.

The Green operator of $V$ for the potential $q$ is $\mathcal{G}_{q}^{V}: \mathcal{C}(V) \longrightarrow \mathcal{C}(V)$ defined for any $f \in \mathcal{C}(V)$ as $\mathcal{G}_{q}^{V}(f)(x)=\sum_{y \in V} G_{q}^{V}(x, y) f(y)$, $x \in V$. Then for any $f \in \mathcal{C}(V), \mathcal{G}_{q}^{V}(f)=\mathcal{G}_{q}^{V}(f-\langle\omega, f\rangle \omega), \mathcal{G}_{q}^{V}$ is self-adjoint and the function $u=\mathcal{G}_{q}^{V}(f) \in \mathcal{C}(V)$ is the unique function in $\omega^{\perp}$ satisfying that $\mathcal{L}_{q}(u)=f-\langle\omega, f\rangle \omega$. Newly, the self-adjointness of $\mathcal{G}_{q}^{V}$ implies that $G_{q}^{V}$ is a symmetric function and the Minimum Principle also implies that $\omega(y) G_{q}^{V}(x, y)<\omega(x) G_{q}^{V}(y, y)$ for any $x, y \in V$, see newly [4].

We remark that the existence and uniqueness of solution for the boundary value problem (3) means that $\mathcal{L}_{q}$ is an automorphism of $\mathcal{C}(F)$ and hence, $\mathcal{G}_{q}^{F}$ is its inverse. Moreover, when $\lambda=0$, then $\mathcal{L}_{q}$ is an automorphism of $\omega^{\perp}$ whose inverse can be extended to $\mathcal{C}(V)$ by considering for any $f \in \mathcal{C}(V)$ its orthogonal component with respect to $\omega$. This extension is precisely $\mathcal{G}_{q}^{V}$ and clearly is singular, since $\mathcal{G}_{q}^{V}(\omega)=0$.

On the other hand, if we label the vertices of $\Gamma$, say $V=\left\{x_{1}, \ldots, x_{n}\right\}$ where $n=|V|$, then each endomorphism of $\mathcal{C}(F)$ can be interpreted as a matrix of order $|F|$. So $\mathcal{L}_{q}$ is identified with the matrix $L_{q}^{V}$ whose diagonal entries are $\kappa\left(x_{j}\right)+q\left(x_{j}\right)$ and whose off-diagonal entries are $-c\left(x_{i}, x_{j}\right), i, j=1, \ldots, n$. Moreover if for a proper subset $F \subset V$, we interpret $\mathcal{L}_{q}$ as an endomorphism of $\mathcal{C}(F)$, then it can be identified with the matrix $L_{q}^{F}$ obtained from $L_{q}^{V}$ by deleting the rows and the columns corresponding to the vertices in $F^{c}$. Notice that, as the potential is real-valued, all the above matrices are real-valued and symmetric.

We also denote by $\mathrm{G}_{q}^{F}$ the matrix identified with the Green operator $\mathcal{G}_{q}^{F}$ defined above. With these identifications, $\mathrm{G}_{q}^{F}=\left(\mathrm{L}_{q}^{F}\right)^{-1}$ when either $F$ is a proper subset of $V$ or $\lambda>0$. Moreover, when $\lambda=0$, then $\mathrm{G}_{q}^{V}=\left(\mathrm{L}_{q}^{V}\right)^{\#}$, the Group Inverse of $\mathrm{L}_{q}^{V}$. Since the group inverse coincides with the inverse when the matrix is invertible, we have that $\mathrm{G}_{q}^{F}=\left(\mathrm{L}_{q}^{F}\right)^{\#}$ for any non-empty subset $F \subset V$ and any $\lambda \geq 0$.

Given a non-empty subset $F \subset V$, an eigenvalue of the boundary problem (3) is $z \in \mathbb{C}$ such that the Schrödinder operator $\mathcal{L}_{q-z}$ is singular on $\mathcal{C}(F ; \mathbb{C})$. Equivalently, $z \in \mathbb{C}$ is an eigenvalue of the boundary problem (3) if there exists $u \in \mathcal{C}(F ; \mathbb{C})$ non-null and such that $\mathcal{L}_{q}(u)=z u$ on $F$. Each $u \in \mathcal{C}(F ; \mathbb{C})$ satisfying the above identity is called eigenfunction of the boundary problem (3) associated with $z$.

Since $q$ is a real-valued potential, any eigenvalue must be real. This claim follows by taking into account that if $u \in \mathcal{C}(F ; \mathbb{C})$ is non null and satisfies that $\mathcal{L}_{q}(u)=z u$ on $F$, then

$$
z\|u\|^{2}=\left\langle\mathcal{L}_{q}(u), u\right\rangle=\left\langle u, \mathcal{L}_{\bar{q}}(u)\right\rangle=\left\langle u, \mathcal{L}_{q}(u)\right\rangle=\bar{z}\|u\|^{2}
$$


which implies $z=\bar{z}$; that is $z \in \mathbb{R}$. On the other hand, if $u, v \in \mathcal{C}(F, \mathbb{C})$ are eigenfunctions corresponding to $z$ and $\hat{z}$ respectively, then

$$
z\langle u, v\rangle=\left\langle\mathcal{L}_{q}(u), v\right\rangle=\left\langle u, \mathcal{L}_{q}(v)\right\rangle=\hat{z}\langle, u, v\rangle
$$

which implies that if $z \neq \hat{z}$, then $\langle u, v\rangle=0$. In particular, if $u \in \mathcal{C}(F, \mathbb{C})$ is an eigenfunction corresponding to $z \in \mathbb{R}$, then $u$ is a real-valued function; that is, $u \in \mathcal{C}(F)$.

If $z \in \mathbb{C}$ is not an eigenvalue of the boundary value problem (3), then $\mathcal{L}_{q-z}$ is an automorphism of $\mathcal{C}(F ; \mathbb{C})$ and then we denote by $\mathcal{G}_{q-z}^{F}$ its inverse. Moreover, if $G_{q-z}^{F}: F \times F \longrightarrow \mathbb{R}$ is given for any $y \in V$ as $G_{q-z}^{F}(\cdot, y)$, the unique solution of the equation $\mathcal{L}_{q}(u)=\varepsilon_{y}$ on $F$, then $\mathcal{G}_{q-z}^{F}(f)(x)=\sum_{y \in F} G_{q-z}^{F}(x, y) f(y)$, for any $f \in \mathcal{C}(F, \mathbb{C})$ and any $x \in F$.

The following result is the discrete version of the well-known Spectral Theorem. Its proof follows the standard reasoning involving the minimization of the energy, so we have Inequality (4) into account.

Theorem 3.1 (Spectral Theorem). For any non-empty subset $F \subset V$, there exist real values $\mu_{1}^{F} \leq \cdots \leq \mu_{|F|}^{F}$ and an orthonormal basis $\left\{v_{j}^{F}\right\}_{j=1}^{|F|} \subset \mathcal{C}(F)$ satisfying the following properties:

(i) $\mathcal{L}_{q}\left(v_{j}^{F}\right)=\mu_{j}^{F} v_{j}^{F}$ on $F, j=1, \ldots,|F|$. Moreover, if $z \in \mathbb{R}$ is an eigenvalue of the boundary value problem (3), then $z=\mu_{j}^{F}$ for some $j=1, \ldots,|F|$.

(ii) $\lambda \leq \mu_{1}^{F}<\mu_{2}^{F}$ and $v_{1}^{F}(x)>0$ for any $x \in F$. Moreover, $\mu_{1}^{F}=\lambda$ iff $F=V$ and then $v_{1}^{F}=\omega$. In particular, $\mu_{1}^{F}>0$, except when $F=V$ and $\lambda=0$, simultaneously.

(iii) For any $u \in \mathcal{C}(F ; \mathbb{C})$ then $\mathcal{L}_{q}(u)(x)=\sum_{j=1}^{|F|} \mu_{j}^{F}\left\langle u, v_{j}^{F}\right\rangle v_{j}^{F}(x)$ for any $x \in F$.

As a very nice consequence of the Spectral Theorem, we can obtain the expression of $G_{q}^{F}(x, y)$, the Green function of $F$ for the potential $q$, in terms of eigenvalues and eigenfunctions of $\mathcal{L}_{q}$. Prior to do this, for any $a \in \mathbb{C}$ we define $a^{\#}=$ $\left\{\begin{array}{cl}a^{-1}, & \text { if } a \neq 0 \\ 0, & \text { if } a=0\end{array}\right.$

Using the same notation as in Theorem 3.1, we get the following result.

Theorem 3.2 (Mercer Theorem). Given a non-empty subset $F \subset V$, then

$$
G_{q}^{F}(x, y)=\sum_{j=1}^{|F|}\left(\mu_{j}^{F}\right)^{\#} v_{j}^{F}(x) v_{j}^{F}(y), \quad x, y \in V .
$$

Moreover, if $z \in \mathbb{C} \backslash\left\{\mu_{1}^{F} \leq \cdots \leq \mu_{|F|}^{F}\right\}$, then

$$
G_{q-z}^{F}(x, y)=\sum_{j=1}^{|F|}\left(\mu_{j}^{F}-z\right)^{-1} v_{j}^{F}(x) v_{j}^{F}(y), \quad x, y \in V .
$$

\section{Schrödinger operators on product networks}

In this section we prove that Schrödinger operators on product network can be expressed in separated variables and hence we can obtain a discrete version of the separation of variables method.

Let us consider two different connected networks $\left(\Gamma_{1}, c_{1}\right)$ and $\left(\Gamma_{2}, c_{2}\right)$ with vertex sets $V_{1}$ and $V_{2}$.

We define the product network as the network $\Gamma=\Gamma_{1} \times \Gamma_{2}=(V, c)$ where $V=V_{1} \times V_{2}$ and the conductance is given by

$$
c\left(\left(x_{1}, y_{1}\right),\left(x_{2}, y_{2}\right)\right)=\left\{\begin{aligned}
c_{1}\left(x_{1}, x_{2}\right), & \text { if } y_{1}=y_{2}, \\
c_{2}\left(y_{1}, y_{2}\right), & \text { if } x_{1}=x_{2}, \\
0, & \text { otherwise }
\end{aligned}\right.
$$

Clearly $\Gamma_{1} \times \Gamma_{2}$ is also connected.

Given $u \in \mathcal{C}\left(V_{1} \times V_{2}\right)$ for any $(x, y) \in V_{1} \times V_{2}, u_{y} \in \mathcal{C}\left(V_{1}\right), u^{x} \in \mathcal{C}\left(V_{2}\right)$ denote the functions defined as $u_{y}(z)=u(z, y)$ for any $z \in V_{1}$ and by $u^{x}(z)=u(x, z)$ for any $z \in V_{2}$.

Given $u \in \mathcal{C}\left(V_{1}\right)$ and $v \in \mathcal{C}\left(V_{2}\right)$ the tensor product of $u$ and $v$ is $u \otimes v \in \mathcal{C}\left(V_{1} \times V_{2}\right)$ defined as $(u \otimes v)(x, y)=u(x) v(y)$ for any $(x, y) \in V_{1} \times V_{2}$. Notice that given two weights $\omega_{i} \in \Omega\left(V_{i}\right), i=1,2$, then $\omega_{1} \otimes \omega_{2} \in \Omega\left(V_{1} \times V_{2}\right)$. Moreover, given $x \in V_{1}$ and $y \in V_{2}$ we have $\varepsilon_{(x, y)}=\varepsilon_{x} \otimes \varepsilon_{y}$.

We denote by $\mathcal{L}^{i}$ the combinatorial Laplacian of the network $\Gamma_{i}, i=1,2$ and by $\mathcal{L}$ the combinatorial Laplacian of the product network $\Gamma_{1} \times \Gamma_{2}$. The following result establishes that the combinatorial Laplacian of a product network can be expressed in separable variables when it operates on a tensor product function. This property justifies the name of separation of variables for the technique to solve boundary value problems on product networks. 
Proposition 4.1. Given $u_{i} \in \mathcal{C}\left(V_{i}\right), i=1,2$ then

$$
\mathcal{L}\left(u_{1} \otimes u_{2}\right)=\mathcal{L}^{1}\left(u_{1}\right) \otimes u_{2}+u_{1} \otimes \mathcal{L}^{2}\left(u_{2}\right) .
$$

In particular, if $\omega_{i} \in \Omega\left(V_{i}\right), i=1,2$, then $q_{\omega_{1} \otimes \omega_{2}}=q_{\omega_{1}}+q_{\omega_{2}}$ and hence, for any $u \in \mathcal{C}\left(V_{1} \times V_{2}\right)$ we have

$$
\mathcal{L}_{q_{\omega_{1} \otimes \omega_{2}}}(u)(x, y)=\mathcal{L}_{q_{\omega_{1}}}^{1}\left(u_{y}\right)(x)+\mathcal{L}_{q_{\omega_{2}}}^{2}\left(u^{x}\right)(y), \quad(x, y) \in V_{1} \times V_{2} .
$$

Proof. Given $u \in \mathcal{C}\left(V_{1} \times V_{2}\right)$ for any $(x, y) \in V_{1} \times V_{2}$ we have that

$$
\begin{aligned}
\mathcal{L}(u)(x, y) & =\sum_{\substack{z \in V_{1} \\
w \in V_{2}}} c((x, y),(z, w))(u(x, y)-u(z, w)) \\
& =\sum_{z \in V_{1}} c_{1}(x, z)(u(x, y)-u(z, y))+\sum_{w \in V_{2}} c_{2}(y, w)(u(x, y)-u(x, w)) \\
& =\mathcal{L}^{1}\left(u_{y}\right)(x)+\mathcal{L}^{2}\left(u^{x}\right)(y) .
\end{aligned}
$$

On the other hand, since $\left(u_{1} \otimes u_{2}\right)_{y}=u_{1} u_{2}(y)$ and $\left(u_{1} \otimes u_{2}\right)^{x}=u_{1}(x) u_{2}$ we obtain that

$$
\mathcal{L}\left(u_{1} \otimes u_{2}\right)(x, y)=u_{2}(y) \mathcal{L}^{1}\left(u_{1}\right)(x)+u_{1}(x) \mathcal{L}^{2}\left(u_{2}\right)(y) .
$$

In particular $\mathcal{L}\left(\omega_{1} \otimes \omega_{2}\right)=\mathcal{L}^{1}\left(\omega_{1}\right) \otimes \omega_{2}+\omega_{1} \otimes \mathcal{L}^{2}\left(\omega_{2}\right)$ and hence,

$$
q_{\omega_{1} \otimes \omega_{2}}=-\left(\omega_{1} \otimes \omega_{2}\right)^{-1} \mathcal{L}\left(\omega_{1} \otimes \omega_{2}\right)=-\omega_{1}^{-1} \mathcal{L}^{1}\left(\omega_{1}\right)-\omega_{2}^{-1} \mathcal{L}^{2}\left(\omega_{2}\right)=q_{\omega_{1}}+q_{\omega_{2}} .
$$

From all above identities we finally obtain that

$$
\begin{aligned}
\mathcal{L}_{q_{\omega_{1} \otimes \omega_{2}}}(u)(x, y) & =\mathcal{L}(u)(x, y)+q_{\omega_{1} \otimes \omega_{2}}(x, y) u(x, y) \\
& =\mathcal{L}^{1}\left(u_{y}\right)(x)+\mathcal{L}^{2}\left(u^{x}\right)(y)+\left(q_{\omega_{1}}(x)+q_{\omega_{2}}(y)\right) u(x, y) \\
& =\mathcal{L}^{1}\left(u_{y}\right)(x)+\mathcal{L}^{2}\left(u^{x}\right)(y)+q_{\omega_{1}}(x) u_{y}(x)+q_{\omega_{2}}(y) u^{x}(y) \\
& =\mathcal{L}_{q_{\omega_{1}}}^{1}\left(u_{y}\right)(x)+\mathcal{L}_{q_{\omega_{2}}(y)}^{2}\left(u^{x}\right)(y) .
\end{aligned}
$$

\section{Boundary value problems on product networks}

As in the preceding section, we consider connected networks $\left(\Gamma_{i}, c_{i}\right)$ with vertex set $V_{i}$ and combinatorial Laplacian $\mathcal{L}^{i}$, $i=1,2$. Then, we also consider the product network $\Gamma_{1} \times \Gamma_{2}$ and its combinatorial Laplacian $\mathcal{L}$.

The boundary value problems we analyze in $\Gamma_{1} \times \Gamma_{2}$, refer to subsets that are also expressed as cartesian products. So, given non empty subsets $F_{i} \subset V_{i}, i=1,2$ we consider $F=F_{1} \times F_{2} \subset V_{1} \times V_{2}$. Then, it is satisfied that

$$
\delta\left(F_{1} \times F_{2}\right)=\left(F_{1} \times \delta\left(F_{2}\right)\right) \cup\left(\delta\left(F_{1}\right) \times F_{2}\right),
$$

where we allow $F_{i}=V_{i}$ in which case $\delta\left(F_{i}\right)=\emptyset, i=1,2$.

Given $\omega_{i} \in \Omega\left(V_{i}\right), i=1,2$ and $\lambda \geq 0$, we consider the real-valued potential $q=q_{\omega_{1} \otimes \omega_{2}}+\lambda$. We are interested in studying the boundary value problem (3) on $F=F_{1} \times F_{2}$ and also in computing the corresponding Green function $\mathcal{G}_{q}^{F}$. To do this, we first split $\lambda$ as $\lambda_{1}+\lambda_{2}$ where $\lambda_{1}, \lambda_{2} \geq 0$ and then apply the Spectral Theorem to the two boundary value problems $\mathcal{L}_{q_{i}}\left(u_{i}\right)=f_{i}$ on $F_{i}$, where $q_{i}=q_{\omega_{i}}+\lambda_{i}$ and $f_{i}, u_{i} \in \mathcal{C}\left(F_{i}\right), i=1$, 2. Specifically, let $\mu_{1}^{F_{i}} \leq \cdots \leq \mu_{\left|F_{i}\right|}^{F_{i}}$ the eigenvalues of the boundary value problem $\mathcal{L}_{q_{i}}\left(u_{i}\right)=f_{i}$ on $F_{i}, i=1,2$ and $\left.\left\{v_{j}^{F_{i}}\right\}_{j=1}\right|_{i} \mid \subset \mathcal{C}\left(F_{i}\right)$ a corresponding orthonormal system of eigenfunctions.

Remember that always $\mu_{1}^{F_{i}}$ is simple and moreover $v_{1}^{F_{i}}>0$ on $F_{i}, i=1$, 2. In addition, $\mu_{1}^{F_{i}}=\lambda_{i}$ iff $F_{i}=V_{i}$ and then $v_{1}^{F_{i}}=\omega_{i}$. Therefore, $\mu_{1}^{F_{i}}>0$, except when $F_{i}=V_{i}$ and $\lambda_{i}=0$, simultaneously.

The main result in product networks is that the eigenvalues and the eigenfunctions for the boundary value problem (3) in product subsets, are completely characterized in terms of the eigenvalues and the eigenfunctions of each factor.

Theorem 5.1. For any $j=1, \ldots,\left|F_{1}\right|$ and any $k=1, \ldots,\left|F_{2}\right|$ we have that

$$
\mathcal{L}_{q}\left(v_{j}^{F_{1}} \otimes v_{k}^{F_{2}}\right)=\left(\mu_{j}^{F_{1}}+\mu_{k}^{F_{2}}\right) v_{j}^{F_{1}} \otimes v_{k}^{F_{2}} \text { on } F_{1} \times F_{2} .
$$

Moreover, $\left\{\mu_{j}^{F_{1}}+\mu_{k}^{F_{2}}\right\}_{\substack{1 \leq j \leq\left|F_{1}\right| \\ 1 \leq k \leq\left|F_{2}\right|}}$ are the eigenvalues of $\mathcal{L}_{q}$ on $F_{1} \times F_{2}$ and the set $\left\{v_{j}^{F_{1}} \otimes v_{k}^{F_{2}}\right\}_{\substack{1 \leq j \leq\left|F_{1}\right| \\ 1 \leq k \leq\left|F_{2}\right|}}$ is an orthonormal basis in $\mathcal{C}\left(F_{1} \times F_{2}\right)$.

Proof. From Proposition 4.1, on $F_{1} \times F_{2}$ we have

$$
\begin{aligned}
\mathcal{L}_{q}\left(v_{j}^{F_{1}} \otimes v_{k}^{F_{2}}\right) & =\mathcal{L}_{q_{1}}^{1}\left(v_{j}^{F_{1}}\right) \otimes v_{k}^{F_{2}}+v_{j}^{F_{1}} \otimes \mathcal{L}_{q_{2}}\left(v_{k}^{F_{2}}\right) \\
& =\mu_{j}^{F_{1}} v_{j}^{F_{1}} \otimes v_{k}^{F_{2}}+\mu_{k}^{F_{2}} v_{j}^{F_{1}} \otimes v_{k}^{F_{2}}=\left(\mu_{j}^{F_{1}}+\mu_{k}^{F_{2}}\right) v_{j}^{F_{1}} \otimes v_{k}^{F_{2}} .
\end{aligned}
$$


Since the system $\left\{v_{j}^{F_{1}} \otimes v_{k}^{F_{2}}\right\}_{\substack{1 \leq j \leq\left|F_{1}\right| \\ 1 \leq k \leq F_{2} \mid}} \subset \mathcal{C}\left(F_{1} \times F_{2}\right)$ is orthonormal and $\operatorname{dim} \mathcal{C}\left(F_{1} \times F_{2}\right)=\left|F_{1}\right| \cdot\left|F_{2}\right|$ we conclude that $\left\{v_{j}^{F_{1}} \otimes v_{k}^{F_{2}}\right\}_{\substack{1 \leq j \leq\left|F_{1}\right| \\ 1 \leq k \leq F_{2} \mid}}$ is a basis of $\mathcal{C}\left(F_{1} \times F_{2}\right)$. Moreover, since any eigenfunction corresponding to an eigenvalue, other than $\mu_{j}^{F_{1}}+\mu_{k}^{F_{2}}, j=1, \ldots,\left|F_{1}\right|, k=1, \ldots,\left|F_{2}\right|$, must be orthogonal to the above basis, we conclude that $\left\{\mu_{j}^{F_{1}}+\mu_{k}^{F_{2}}\right\}_{\substack{1 \leq j \leq\left|F_{1}\right| \\ 1 \leq k \leq\left|F_{2}\right|}}$ determines all eigenvalues.

Notice that $\mu_{1}^{F_{1}}+\mu_{1}^{F_{2}}$ is the lowest eigenvalue, it is simple and moreover $v_{1}^{F_{1}} \otimes v_{1}^{F_{2}}>0$ on $F_{1} \times F_{2}$. In addition, since $\mu_{j}^{F_{1}}+\mu_{k}^{F_{2}} \geq \lambda_{1}+\lambda_{2}=\lambda \geq 0$ we have that $\mu_{j}^{F_{1}}+\mu_{k}^{F_{2}}>0$ except when $F_{1}=V_{1}, F_{2}=V_{2}$ and $\lambda=0$ simultaneously. Notice that $\lambda=0$ iff $\lambda_{1}=\lambda_{2}=0$ and then, if in addition $F_{1}=V_{1}, F_{2}=V_{2}$, we have $v_{1}^{F_{i}}=\omega_{i}, i=1,2$.

In general, the eigenvalues $\mu_{j}^{F_{1}}+\mu_{k}^{F_{2}}, j=1, \ldots,\left|F_{1}\right|, k=1, \ldots,\left|F_{2}\right|$ when $j+k>2$ have multiplicity greater than 1, even if each factor has only simple eigenvalues. For instance this happens in the square network $\Gamma \times \Gamma$ for the weight $\omega \otimes \omega, \omega \in \Omega$. In this case, given $F \subset V$, each eigenvalue of the boundary value problem (3) on $F \times F$ other than the $2 \mu_{j}^{F}$, $j=1, \ldots,|F|$, has multiplicity 2 at least: Given $j \neq k$, then $v_{j}^{F} \otimes v_{k}^{F}$ and $v_{k}^{F} \otimes v_{j}^{F}$ are eigenfunctions corresponding to $\mu_{j}^{F}+\mu_{k}^{F}$.

The main consequence of Theorem 5.1 is that we can compute the Green function for product networks in terms of the eigenvalues and the eigenfunctions of each factor by applying the Mercer Theorem.

Corollary 5.2. Under hypothesis of Theorem 5.1, for any $\left(x_{1}, y_{1}\right),\left(x_{2}, y_{2}\right) \in V_{1} \times V_{2}$, we have that

$$
G_{q}^{F_{1} \times F_{2}}\left(\left(x_{1}, y_{1}\right),\left(x_{2}, y_{2}\right)\right)=\sum_{j=1}^{\left|F_{1}\right|} \sum_{k=1}^{\left|F_{2}\right|}\left(\mu_{j}^{F_{1}}+\mu_{k}^{F_{2}}\right)^{\#} v_{j}^{F_{1}}\left(x_{1}\right) v_{j}^{F_{1}}\left(x_{2}\right) v_{k}^{F_{2}}\left(y_{1}\right) v_{k}^{F_{2}}\left(y_{2}\right)
$$

The above formula requires the knowledge of eigenvalues and eigenfunctions for the two factors. Therefore, except for structured networks, the application of the above method is very restrictive. F. Chung and S.T. Yau obtained in [11], see also [13,14], a nice formula based in a clever use of the complex integration, that avoids the computation of eigenvalues and eigenvectors and only needs the evaluation of the Green function of each factor, but considering complex-valued potentials. Although the above authors only consider normalized Laplacians on regular networks, and hence combinatorial Laplacians, their technique is easily extensible to positive semidefinite Schrödinger operators.

Lemma 5.3. Let $a, b \in \mathbb{C}$ and consider $\gamma$ a smooth and simple curve enclosing $a$ and moreover it leaves $-b$ in its exterior when $a+b \neq 0$. Then,

$$
(a+b)^{\#}=\frac{1}{2 \pi i} \int_{\gamma} \frac{d z}{(a-z)(b+z)}
$$

Proof. If $f(z)=\frac{1}{(a-z)(b+z)}$, then $f$ has isolated singularities at $a$ and $-b$ and moreover, since $f(z)=\frac{1}{a+b}\left[\frac{1}{a-z}+\right.$ $\left.\frac{1}{b+z}\right]$ when $a+b \neq 0$ and $f(z)=\frac{-1}{(z-a)^{2}}$ when $a+b=0$, the residue of $f$ at $a$ is $(a+b)^{\#}$. Therefore, we obtain the result applying the Residue Theorem, see [12].

We can use the above identity, to express à la Chung \& Yau, the Green function of the boundary value problem (3) in a product set.

Proposition 5.4. In the preceding conditions, for any $\left(x_{1}, y_{1}\right),\left(x_{2}, y_{2}\right) \in V_{1} \times V_{2}$, we have that

$$
G_{q}^{F_{1} \times F_{2}}\left(\left(x_{1}, y_{1}\right),\left(x_{2}, y_{2}\right)\right)=\frac{1}{2 \pi i} \int_{\gamma} G_{q_{1}+z}^{F_{1}}\left(x_{1}, x_{2}\right) G_{q_{2}-z}^{F_{2}}\left(y_{1}, y_{2}\right) d z
$$

where $\gamma$ is a smooth and simple curve satisfying the following conditions:

(i) If either $\lambda>0$ or $F_{1} \times F_{2} \neq V_{1} \times V_{2}$, then $\gamma$ surrounds $\mu_{1}^{F_{1}}, \ldots, \mu_{\left|F_{1}\right|}^{F_{1}}$ and leave $-\mu_{1}^{F_{2}}, \ldots,-\mu_{\left|F_{2}\right|}^{F_{2}}$ in its exterior.

(ii) If $\lambda=0, F_{1}=V_{1}$ and $F_{2}=V_{2}$, then $\gamma$ surrounds $\mu_{1}^{F_{1}}, \ldots, \mu_{\left|F_{1}\right|}^{F_{1}}$ and leave $-\mu_{2}^{F_{2}}, \ldots,-\mu_{\left|F_{2}\right|}^{F_{2}}$ in its exterior.

Proof. From Corollary 5.2 and applying Lemma 5.3, we have that

$$
\begin{aligned}
G_{q}^{F_{1} \times F_{2}}\left(\left(x_{1}, y_{1}\right),\left(x_{2}, y_{2}\right)\right) & =\frac{1}{2 \pi i} \int_{\gamma}\left[\sum_{j=1}^{\left|F_{1}\right|} \sum_{k=1}^{\left|F_{2}\right|} \frac{v_{j}^{F_{1}}\left(x_{1}\right) v_{j}^{F_{1}}\left(x_{2}\right) v_{k}^{F_{2}}\left(y_{1}\right) v_{k}^{F_{2}}\left(y_{2}\right)}{\left(\mu_{j}^{F_{1}}-z\right)\left(\mu_{k}^{F_{2}}+z\right)}\right] d z \\
& =\frac{1}{2 \pi i} \int_{\gamma}\left[\sum_{j=1}^{\left|F_{1}\right|} \frac{v_{j}^{F_{1}}\left(x_{1}\right) v_{j}^{F_{1}}\left(x_{2}\right)}{\mu_{j}^{F_{1}}-z} \sum_{k=1}^{\left|F_{2}\right|} \frac{v_{k}^{F_{2}}\left(y_{1}\right) v_{k}^{F_{2}}\left(y_{2}\right)}{\mu_{k}^{F_{2}}+z}\right] d z
\end{aligned}
$$


and the result follows by applying the second part of Mercer Theorem and taking into account that, according to the definition of $\gamma$, any complex value $z \in \mathbb{C}$ lying on the trace of $\gamma$, is neither an eigenvalue for the boundary value problem (3) on $F_{1}$ nor $-z$ is an eigenvalue for the boundary value problem (3) on $F_{2}$.

Although Chung \& Yau's method avoids the explicit computation of eigenvalues and eigenfunctions, it requires to calculate an infinite family of Green's functions, depending on a complex parameter, and hence to evaluate a complex integral. We finish this paper showing a technique that mix the two former methods. It only requires the computation of eigenvalues and eigenfunctions for one of the factor networks and also the computation of a finite family of Green's functions corresponding to the other product network. In fact this method is nothing else but the discrete version of the well-known Separation of Variables Method to solve boundary value problems in PDE.

The key issue to apply the Separation of Variables Method lies on the use of an appropriate expression for functions in $\mathcal{C}\left(F_{1} \times F_{2}\right)$. With the above notations, for any given $h \in \mathcal{C}\left(F_{1} \times F_{2}\right)$, for any $j=1, \ldots,\left|F_{1}\right|$ and any $k=1, \ldots,\left|F_{2}\right|$ we consider the functions $\alpha_{j}(h) \in \mathcal{C}\left(F_{2}\right)$ and $\beta_{k}(h) \in \mathcal{C}\left(F_{1}\right)$ defined as

$$
\begin{aligned}
& \alpha_{j}(h)(y)=\left\langle h_{y}, v_{j}^{F_{1}}\right\rangle=\sum_{z \in V_{1}} h(z, y) v_{j}^{F_{1}}(z)=\sum_{z \in F_{1}} h(z, y) v_{j}^{F_{1}}(z), \quad y \in V_{2}, \\
& \beta_{k}(h)(x)=\left\langle h^{x}, v_{k}^{F_{2}}\right\rangle=\sum_{z \in V_{2}} h(x, z) v_{k}^{F_{2}}(z)=\sum_{z \in F_{2}} h(x, z) v_{k}^{F_{2}}(z), \quad x \in V_{1} .
\end{aligned}
$$

Lemma 5.5. For any $h \in \mathcal{C}\left(F_{1} \times F_{2}\right)$ the following identities hold

$$
h=\sum_{j=1}^{\left|F_{1}\right|} v_{j}^{F_{1}} \otimes \alpha_{j}(h)=\sum_{k=1}^{\left|F_{2}\right|} \beta_{k}(h) \otimes v_{k}^{F_{2}}
$$

In particular, if $\hat{h} \in \mathcal{C}\left(F_{1} \times F_{2}\right)$, then $h=\hat{h}$ iff $\alpha_{j}(h)=\alpha_{j}(\hat{h}), j=1, \ldots,\left|F_{1}\right|$ or equivalently iff $\beta_{k}(h)=\beta_{k}(\hat{h}), k=1, \ldots,\left|F_{2}\right|$.

Proof. Since $\left\{v_{k}^{F_{2}}\right\}_{1 \leq k \leq\left|F_{2}\right|}$ is an orthonormal basis in $\mathcal{C}\left(F_{2}\right)$, for any $x \in F_{1}$ we have that $h^{x}=\sum_{k=1}^{\left|F_{2}\right|}\left\langle h^{x}, v_{k}^{F_{2}}\right\rangle v_{k}^{F_{2}}=$ $\sum_{k=1}^{\left|F_{2}\right|} \beta(h)(x) v_{k}^{F_{2}}$; that is, for any $y \in F_{2}$ we have

$$
h(x, y)=\sum_{k=1}^{\left|F_{2}\right|} \beta_{k}(h)(x) v_{k}^{F_{2}}(y)=\sum_{k=1}^{\left|F_{2}\right|}\left(\beta_{k}(h) \otimes v_{k}^{F_{2}}\right)(x, y) .
$$

The other identity can be proved in an analogous way.

Theorem 5.6. Under the conditions and notations in this section, for $i=1,2$ consider the real-valued potentials $p_{k}^{1}=q_{1}+\mu_{k}^{F_{2}}=$ $q_{\omega_{1}}+\lambda_{1}+\mu_{k}^{F_{2}} \in \mathcal{C}\left(F_{1}\right), k=1, \ldots,\left|F_{2}\right|$ and $p_{j}^{2}=q_{2}+\mu_{j}^{F_{1}}=q_{\omega_{2}}+\lambda_{2}+\mu_{j}^{F_{1}} \in \mathcal{C}\left(F_{2}\right), j=1, \ldots,\left|F_{1}\right|$. Then,

$$
\begin{aligned}
G_{q}^{F_{1} \times F_{2}}\left(\left(x_{1}, y_{1}\right),\left(x_{2}, y_{2}\right)\right) & =\sum_{\substack{k=1 \\
\left|F_{1}\right|}}^{\left|F_{2}\right|} G_{p_{k}^{1}}^{F_{1}}\left(x_{1}, x_{2}\right) v_{k}^{F_{2}}\left(y_{1}\right) v_{k}^{F_{2}}\left(y_{2}\right) \\
& =\sum_{j=1}^{F_{2}} G_{p_{j}^{2}}^{F_{1}}\left(y_{1}, y_{2}\right) v_{j}^{F_{1}}\left(x_{1}\right) v_{j}^{F_{1}}\left(x_{2}\right) .
\end{aligned}
$$

Proof. Because the proof of both identities follows the same reasoning, we only prove the first one. Moreover, we first develop the separation of variables technique in a general setting and then we specify it to the computation of the Green function.

Given $f, u \in \mathcal{C}\left(F_{1} \times F_{2}\right)$, applying Lemma 5.5 we have

$$
f=\sum_{k=1}^{\left|F_{2}\right|} \beta_{k}(f) \otimes v_{k}^{F_{2}} \text { and } u=\sum_{k=1}^{\left|F_{2}\right|} \beta_{k}(u) \otimes v_{k}^{F_{2}} .
$$

On the other hand, from Proposition 4.1, we have that

$$
\begin{aligned}
\mathcal{L}_{q}(u) & =\sum_{k=1}^{\left|F_{2}\right|} \mathcal{L}_{q_{1}}^{1}\left(\beta_{k}(u)\right) \otimes v_{k}^{F_{2}}+\sum_{k=1}^{\left|F_{2}\right|} \mu_{k}^{F_{2}} \beta_{k}(u) \otimes v_{k}^{F_{2}} \\
& =\sum_{k=1}^{\left|F_{2}\right|}\left[\mathcal{L}_{q_{1}}^{1}\left(\beta_{k}(u)\right)+\mu_{k}^{F_{2}} \beta_{k}(u)\right] \otimes v_{k}^{F_{2}}=\sum_{k=1}^{\left|F_{2}\right|} \mathcal{L}_{p_{k}}^{1}\left(\beta_{k}(u)\right) \otimes v_{k}^{F_{2}}
\end{aligned}
$$

and hence, $f=\mathcal{L}_{q}(u)$ on $F_{1} \times F_{2}$ iff

$$
\mathcal{L}_{p_{k}^{1}}^{1}\left(\beta_{k}(u)\right)=\beta_{k}(f), \quad \text { on } F_{1} \quad k=1, \ldots,\left|F_{2}\right| .
$$


To analyze the above boundary value problem, we first observe that $p_{k}^{1}=q_{\omega_{1}}+\lambda_{1}+\mu_{k}^{F_{2}}, k=1, \ldots,\left|F_{2}\right|$.

When either $F_{1} \times F_{2} \neq V_{1} \times V_{2}$ or $\lambda_{1}+\lambda_{2}=\lambda>0$, then $\lambda_{1}+\mu_{k}^{F_{2}}>0, k=1, \ldots,\left|F_{2}\right|$, and hence all the above boundary value problems have a unique solution that is given by

$$
\beta_{k}(u)(x)=\sum_{z \in V_{1}} G_{p_{k}^{1}}^{F_{1}}(x, z) \beta_{k}(f)(z)
$$

When $F_{i}=V_{i}, i=1,2$ and $\lambda=0$ then the boundary value problem $\mathcal{L}_{q}(u)=f$ on $V_{1} \times V_{2}$ is solvable iff $f \in\left(\omega_{1} \otimes \omega_{2}\right)^{\perp}$, and then, there exists a unique solution in $\left(\omega_{1} \otimes \omega_{2}\right)^{\perp}$. In this case $\lambda_{1}=\lambda_{2}=0, \mu_{1}^{F_{2}}=0$ and hence $\lambda_{1}+\mu_{k}^{F_{2}}=\mu_{k}^{F_{2}}>0$, $k=2, \ldots,\left|F_{2}\right|$.

Moreover, $\left\langle\beta_{1}(f), \omega_{1}\right\rangle=\left\langle f, \omega_{1} \otimes \omega_{2}\right\rangle$ since $v_{1}^{F_{2}}=\omega_{2}$ and hence we have that $f \in\left(\omega_{1} \otimes \omega_{2}\right)^{\perp}$ iff $\beta_{1}(f) \in \omega_{1}^{\perp}$. So, under the above conditions, if $f \in\left(\omega_{1} \otimes \omega_{2}\right)^{\perp}$ all the boundary value problems $\mathcal{L}_{p_{k}^{1}}^{1}\left(\beta_{k}(u)\right)=\beta_{k}(f)$ on $F_{1}, k=2, \ldots,\left|F_{2}\right|$ have

$$
\beta_{k}(u)(x)=\sum_{z \in V_{1}} G_{p_{k}^{1}}^{F_{1}}(x, z) \beta_{k}(f)(z)
$$

as unique solution, whereas

$$
\beta_{1}(u)(x)=\sum_{z \in V_{1}} G_{p_{1}^{1}}^{F_{1}}(x, y) \beta_{1}(f)(z)
$$

is the unique solution of the boundary value problem $\mathcal{L}_{p_{1}^{1}}^{1}\left(\beta_{1}(u)\right)=\beta_{1}(f)$ belonging to $\omega_{1}^{\perp}$.

Consider now $x_{2} \in F_{1}$ and $y_{2} \in F_{2}$. Then $f=\varepsilon_{\left(x_{2}, y_{2}\right)}=\varepsilon_{x_{2}} \otimes \varepsilon_{y_{2}}$ and hence,

$$
\beta_{k}(f)=v_{k}^{F_{2}}\left(y_{2}\right) \varepsilon_{x_{2}}, \quad k=1, \ldots,\left|F_{2}\right| \text {. }
$$

When either $F_{1} \times F_{2} \neq V_{1} \times V_{2}$ or $\lambda>0$, for any $k=1, \ldots,\left|F_{2}\right|$, we have

$$
\beta_{k}(u)(x)=\sum_{z \in V_{1}} G_{p_{k}^{1}}^{F_{1}}(x, z) \beta_{k}(f)(z)=G_{p_{k}^{1}}^{F_{1}}\left(x, x_{2}\right) v_{k}^{F_{2}}\left(y_{2}\right), \quad x \in V_{1},
$$

which implies that

$$
\begin{aligned}
G_{q}^{F_{1} \times F_{2}}\left(\left(x_{1}, y_{1}\right),\left(x_{2}, y_{2}\right)\right) & =\sum_{k=1}^{\left|F_{2}\right|} \beta_{k}(u)\left(x_{1}\right) v_{k}^{F_{2}}\left(y_{1}\right) \\
& =\sum_{k=1}^{\left|F_{2}\right|} G_{p_{k}^{1}}^{F_{1}}\left(x_{1}, x_{2}\right) v_{k}^{F_{2}}\left(y_{2}\right) v_{k}^{F_{2}}\left(y_{1}\right) .
\end{aligned}
$$

When $F_{i}=V_{i}, i=1,2$ and $\lambda=0$, since $v_{1}^{F_{i}}=\omega_{i}$, we consider now $f=\varepsilon_{\left(x_{2}, y_{2}\right)}-\omega_{1}\left(x_{2}\right) \omega_{2}\left(y_{2}\right)\left(\omega_{1} \otimes \omega_{2}\right)=$ $\varepsilon_{x_{2}} \otimes \varepsilon_{y_{2}}-\omega_{1}\left(x_{2}\right) \omega_{2}\left(y_{2}\right)\left(\omega_{1} \otimes \omega_{2}\right)$. Then,

$$
\beta_{1}(f)=\omega_{2}\left(y_{2}\right)\left[\varepsilon_{x_{2}}-\omega_{1}\left(x_{2}\right) \omega_{1}\right], \quad \beta_{k}(f)=v_{k}^{F_{2}}\left(y_{2}\right) \varepsilon_{x_{2}}, \quad k=2, \ldots,\left|F_{2}\right|
$$

which implies that

$$
\beta_{k}(u)(x)=\sum_{z \in V_{1}} G_{p_{k}^{1}}^{F_{1}}(x, z) \beta_{k}(f)(z)=G_{p_{k}^{1}}^{F_{1}}\left(x, x_{2}\right) v_{k}^{F_{2}}\left(y_{2}\right), \quad x \in V_{1}
$$

where we have taken into account that $p_{1}=q_{\omega_{1}}$ and that

$$
\sum_{z \in V_{1}} G_{p_{1}^{1}}^{F_{1}}(x, z) \omega_{1}(z)=\mathcal{G}_{q_{\omega_{1}}}^{F_{1}}\left(\omega_{1}\right)=0 .
$$

Therefore, the result follows as in the above case.

\section{The Green function of $P_{m} \times S_{h}$}

As an illustration of the main result of the paper, we compute the Green function of a positive semidefinite Schrödinger of the cartesian product of two networks: a path $P_{m}$ and a Star network $S_{h}$. Notice that none of the factors is regular as it was required in previous works, see for instance [14]. In this case, we are going to apply Theorem 5.6 by using the eigenvalues and eigenfunctions of the path and the Green function of the Star. The product network $P_{5} \times S_{3}$ is depicted in Fig. 3 and its factors in Fig. 2.

Consider $P_{m}$ the path on $m$ vertices labeled $V_{1}=\left\{x_{1}, \ldots, x_{m}\right\}$ and constant conductance $c\left(x_{i}, x_{i+1}\right)=c$ for $i=1, \ldots, m-1$ and $c>0$. It is known that, the eigenvalues and eigenfunctions of a path $P_{m}$ for $\mathcal{L}$ are, see [6],

$$
\mu_{j}=2 c\left(1-r_{j}\right)
$$




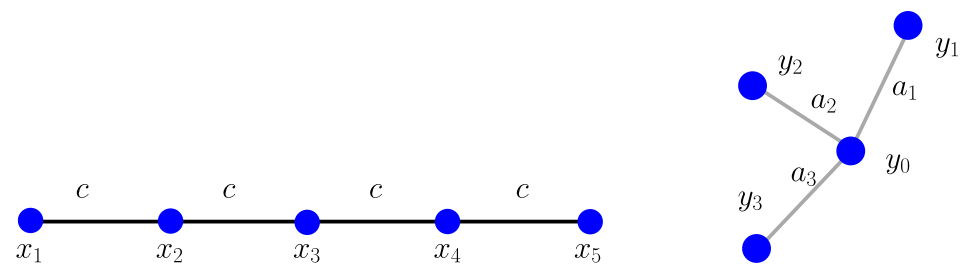

Fig. 2. A path $P_{5}$ (left) and a Star $S_{3}$ (right).

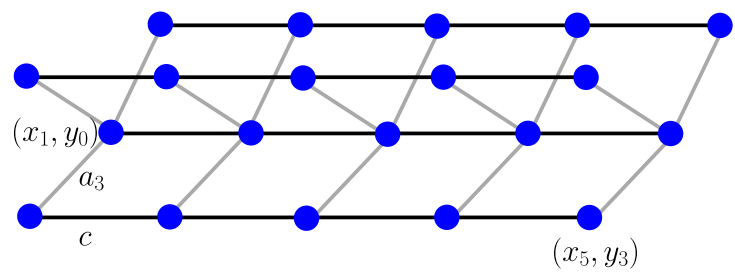

Fig. 3. The product network $P_{5} \times S_{3}$.

where $r_{j}=\cos \left(\frac{j \pi}{m}\right), 0 \leq j \leq m-1$, and the orthonormal eigenfunctions are

$$
u_{0}\left(x_{k}\right)=\frac{1}{\sqrt{m}}, \quad u_{j}\left(x_{k}\right)=\sqrt{\frac{2}{m}} \cos \left(\frac{(2 k-1) j \pi}{2 m}\right), \quad k=1, \ldots, m .
$$

The Star network $S_{h}$ has $h+1$ vertices, $V_{2}=\left\{y_{0}, y_{1}, \ldots, y_{h}\right\}$, and non null conductances $a_{i}=c\left(y_{i}, y_{0}\right)>0, i=1, \ldots, h$. Moreover, let $\omega_{i}=\omega\left(y_{i}\right), i=0, \ldots, h$ be a weight on $S_{h}$. In addition, given $\lambda_{2} \geq 0$, and the potential $q_{2}=q_{\omega}+\lambda_{2}$ we also consider the corresponding positive semi-definite Schrödinger operator $\mathcal{L}_{q_{2}}$. For the sake of simplicity we consider the following value

$$
Q\left(\lambda_{2}, \omega\right)=\sum_{j=1}^{h} \frac{\omega_{j}^{3}}{\lambda_{2} \omega_{j}+a_{j} \omega_{0}} .
$$

In particular, for $\lambda_{2}=0, Q(\omega)=\frac{1}{\omega_{0}} \sum_{j=1}^{h} \frac{\omega_{j}^{3}}{a_{j}}$.

It is known that if $\lambda_{2}>0$, see [8, Corollary 5.4], the Green function $G_{q_{2}}^{V_{2}}\left(y_{k}, y_{s}\right)$ is given by

$$
\begin{aligned}
G_{q_{2}}^{V_{2}}\left(y_{0}, y_{0}\right) & =\frac{\omega_{0}^{2}}{\lambda_{2}\left[1-\lambda_{2} Q\left(\lambda_{2}, \omega\right)\right]}, \\
G_{q_{2}}^{V_{2}}\left(y_{0}, y_{i}\right) & =\frac{a_{i} \omega_{i} \omega_{0}^{2}}{\lambda_{2}\left[1-\lambda_{2} Q\left(\lambda_{2}, \omega\right)\right]\left[\lambda_{2} \omega_{i}+a_{i} \omega_{0}\right]}, \\
G_{q_{2}}^{V_{2}}\left(y_{k}, y_{i}\right) & =\frac{a_{i} a_{k} \omega_{i} \omega_{k} \omega_{0}^{2}}{\lambda_{2}\left[1-\lambda_{2} Q\left(\lambda_{2}, \omega\right)\right]\left[\lambda_{2} \omega_{i}+a_{i} \omega_{0}\right]\left[\lambda_{2} \omega_{k}+a_{k} \omega_{0}\right]}, \\
G_{q_{2}}^{V_{2}}\left(y_{i}, y_{i}\right) & =\frac{a_{i}^{2} \omega_{i}^{2} \omega_{0}^{2}}{\lambda_{2}\left[1-\lambda_{2} Q\left(\lambda_{2}, \omega\right)\right]\left[\lambda_{2} \omega_{i}+a_{i} \omega_{0}\right]^{2}}+\frac{\omega_{i}}{\lambda_{2} \omega_{i}+a_{i} \omega_{0}},
\end{aligned}
$$

where $i, k=1, \ldots, h$ and $k \neq i$. Moreover, for $\lambda_{2}=0$, the Green function of the Star, with respect to $\omega$ is given by

$$
\begin{aligned}
G_{q_{\omega}}^{V_{2}}\left(y_{0}, y_{0}\right) & =\omega_{0}^{2} Q(\omega), \quad G_{q_{\omega}}^{V_{2}}\left(y_{0}, y_{i}\right)=\omega_{i}\left[\omega_{0} Q(\omega)-\frac{\omega_{i}}{a_{i}}\right], \\
G_{q_{\omega}}^{V_{2}}\left(y_{k}, y_{i}\right) & =\frac{\omega_{i} \omega_{k}}{\omega_{0}}\left[\omega_{0} Q(\omega)-\frac{\omega_{i}}{a_{i}}-\frac{\omega_{k}}{a_{k}}\right], \\
G_{q_{\omega}}^{V_{2}}\left(y_{i}, y_{i}\right) & =\frac{\omega_{i}^{2}}{\omega_{0}}\left[\omega_{0} Q(\omega)-\frac{2 \omega_{i}}{a_{i}}\right]+\frac{\omega_{i}}{a_{i} \omega_{0}},
\end{aligned}
$$

where $i, k=1, \ldots, h$ and $k \neq i$. 
We are now going to obtain the Green function of the product network $P_{m} \times S_{h}$ for the Schrödinger operator $\mathcal{L}_{q}$, where $q=q_{1 \otimes \omega}+\lambda, \lambda>0$. Moreover, we consider that $\lambda_{1}=0$ and $\lambda=\lambda_{2}$. Under the conditions and notations of Section 5 we get the following result, where for the sake of simplicity we denote $G_{q}^{V_{1} \times V_{2}}$ by $G_{q}$.

Theorem 6.1. For $\lambda>0$, and the real-valued potentials $p_{j}=q_{\omega}+\lambda_{j} \in \mathcal{C}\left(V\left(S_{h}\right)\right)$, where $\lambda_{j}=\lambda+4 c \sin ^{2}\left(\frac{j \pi}{2 m}\right)$, $j=0, \ldots, m-1$, the Green function for $P_{m} \times S_{h}$ is

$$
\begin{aligned}
& G_{q}\left(\left(x_{i}, y_{0}\right),\left(x_{k}, y_{0}\right)\right)=\widehat{Q}(\lambda, \omega)+2 \sum_{j=1}^{m-1} \widehat{Q}\left(\lambda_{j}, \omega\right) \cos \left(\frac{(2 i-1) j \pi}{2 m}\right) \cos \left(\frac{(2 k-1) j \pi}{2 m}\right), \\
& G_{q}\left(\left(x_{i}, y_{0}\right),\left(x_{k}, y_{s}\right)\right)=\frac{a_{s} \omega_{s} \widehat{Q}(\lambda, \omega)}{\lambda \omega_{s}+a_{s} \omega_{0}} \\
& +2 a_{s} \omega_{s} \sum_{j=1}^{m-1} \frac{\widehat{Q}\left(\lambda_{j}, \omega\right) \cos \left(\frac{(2 i-1) j \pi}{2 m}\right) \cos \left(\frac{(2 k-1) j \pi}{2 m}\right)}{\lambda_{j} \omega_{s}+a_{s} \omega_{0}}, \\
& G_{q}\left(\left(x_{i}, y_{r}\right),\left(x_{k}, y_{s}\right)\right)=\frac{a_{r} a_{s} \omega_{r} \omega_{s} \widehat{Q}(\lambda, \omega)}{\left[\lambda \omega_{r}+a_{r} \omega_{0}\right]\left[\lambda \omega_{s}+a_{s} \omega_{0}\right]} \\
& +2 a_{r} a_{s} \omega_{r} \omega_{s} \sum_{j=1}^{m-1} \frac{\widehat{Q}\left(\lambda_{j}, \omega\right) \cos \left(\frac{(2 i-1) j \pi}{2 m}\right) \cos \left(\frac{(2 k-1) j \pi}{2 m}\right)}{\left[\lambda_{j} \omega_{r}+a_{r} \omega_{0}\right]\left[\lambda_{j} \omega_{s}+a_{s} \omega_{0}\right]}, \\
& G_{q}\left(\left(x_{i}, y_{r}\right),\left(x_{k}, y_{r}\right)\right)=\frac{a_{r}^{2} \omega_{r}^{2} \widehat{Q}(\lambda, \omega)}{\left[\lambda \omega_{r}+a_{r} \omega_{0}\right]^{2}}+\frac{\omega_{r}}{m\left[\lambda \omega_{r}+a_{r} \omega_{0}\right]} \\
& +2 a_{r}^{2} \omega_{r}^{2} \sum_{j=1}^{m-1} \frac{\widehat{Q}\left(\lambda_{j}, \omega\right) \cos \left(\frac{(2 i-1) j \pi}{2 m}\right) \cos \left(\frac{(2 k-1) j \pi}{2 m}\right)}{\left[\lambda_{j} \omega_{r}+a_{r} \omega_{0}\right]^{2}} \\
& +\frac{2 \omega_{r}}{m} \sum_{j=1}^{m-1} \frac{\cos \left(\frac{(2 i-1) j \pi}{2 m}\right) \cos \left(\frac{(2 k-1) j \pi}{2 m}\right)}{\lambda_{j} \omega_{r}+a_{r} \omega_{0}}
\end{aligned}
$$

for any $i, k=1, \ldots, m, r, s=1, \ldots, h$ and $r \neq s$, where $\widehat{Q}(\lambda, \omega)=\frac{\omega_{0}^{2}}{m \lambda[1-\lambda Q(\lambda, \omega)]}$.

Proof. From Theorem 5.6, we get that

$$
\begin{aligned}
G_{q}\left(\left(x_{i}, y_{r}\right),\left(x_{k}, y_{s}\right)\right) & =\sum_{j=0}^{m-1} G_{p_{j}}^{V_{2}}\left(y_{r}, y_{s}\right) u_{j}\left(x_{i}\right) u_{j}\left(x_{k}\right)=\frac{1}{m} G_{p_{0}}^{V_{2}}\left(y_{r}, y_{s}\right) \\
& +\frac{2}{m} \sum_{j=1}^{m-1} G_{p_{j}}^{V_{2}}\left(y_{r}, y_{s}\right) \cos \left(\frac{(2 i-1) j \pi}{2 m}\right) \cos \left(\frac{(2 k-1) j \pi}{2 m}\right) .
\end{aligned}
$$

The result follows by substituting the values for $G_{p_{j}}^{V_{2}}\left(y_{r}, y_{s}\right)$ given in (7).

Finally, we study the case $\lambda=0$.

Theorem 6.2. For $\lambda=0$, and the real-valued potentials $p_{j}=q_{\omega}+\lambda_{j} \in \mathcal{C}\left(V\left(S_{h}\right)\right)$, where $\lambda_{j}=4 c \sin ^{2}\left(\frac{j \pi}{2 m}\right), j=0, \ldots, m-1$, the Green function for $P_{m} \times S_{h}$ is

$$
\begin{aligned}
& G_{q_{\omega}}\left(\left(x_{i}, y_{0}\right),\left(x_{k}, y_{0}\right)\right)=\frac{\omega_{0}^{2}}{m} Q(\omega)+2 \sum_{j=1}^{m-1} \widehat{Q}\left(\lambda_{j}, \omega\right) \cos \left(\frac{(2 i-1) j \pi}{2 m}\right) \cos \left(\frac{(2 k-1) j \pi}{2 m}\right), \\
& G_{q_{\omega}}\left(\left(x_{i}, y_{0}\right),\left(x_{k}, y_{s}\right)\right)=\frac{\omega_{s}}{m}\left[\omega_{0} Q(\omega)-\frac{\omega_{s}}{a_{s}}\right]
\end{aligned}
$$

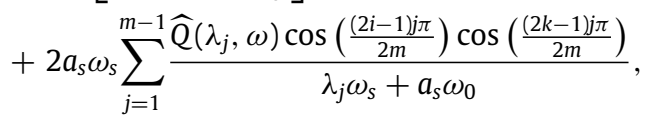




$$
\begin{aligned}
G_{q_{\omega}}\left(\left(x_{i}, y_{r}\right),\left(x_{k}, y_{s}\right)\right) & =\frac{\omega_{r} \omega_{s}}{m \omega_{0}}\left[\omega_{0} Q(\omega)-\frac{\omega_{r}}{a_{r}}-\frac{\omega_{s}}{a_{s}}\right] \\
& +2 a_{r} a_{s} \omega_{r} \omega_{s} \sum_{j=1}^{m-1} \frac{\widehat{Q}\left(\lambda_{j}, \omega\right) \cos \left(\frac{(2 i-1) j \pi}{2 m}\right) \cos \left(\frac{(2 k-1) j \pi}{2 m}\right)}{\left[\lambda_{j} \omega_{r}+a_{r} \omega_{0}\right]\left[\lambda_{j} \omega_{s}+a_{s} \omega_{0}\right]}, \\
G_{q_{\omega}}\left(\left(x_{i}, y_{r}\right),\left(x_{k}, y_{r}\right)\right) & =\frac{\omega_{r}^{2}}{m \omega_{0}}\left[\omega_{0} Q(\omega)-\frac{2 \omega_{r}}{a_{r}}\right]+\frac{\omega_{r}}{m a_{r} \omega_{0}} \\
& +2 a_{r}^{2} \omega_{r}^{2} \sum_{j=1}^{m-1} \frac{\widehat{Q}\left(\lambda_{j}, \omega\right) \cos \left(\frac{(2 i-1) j \pi}{2 m}\right) \cos \left(\frac{(2 k-1) j \pi}{2 m}\right)}{\left[\lambda_{j} \omega_{r}+a_{r} \omega_{0}\right]^{2}} \\
& +\frac{2 \omega_{r}}{m} \sum_{j=1}^{m-1} \frac{\cos \left(\frac{(2 i-1) j \pi}{2 m}\right) \cos \left(\frac{(2 k-1) j \pi}{2 m}\right)}{\lambda_{j} \omega_{r}+a_{r} \omega_{0}},
\end{aligned}
$$

for any $i, k=1, \ldots, m, r, s=1, \ldots, h$ and $r \neq s$.

As far as the authors knowledge neither the eigenvalues nor the eigenfunction of a general Schrödinger operator for the Star network are known. Therefore, the above developments are the only way of obtaining an explicit expression of the Green function of the product network $P_{m} \times S_{h}$. Even in the most simple case; that is, constant weight and conductances in the Star network, the orthonormal basis of eigenfunctions is still quite intricate.

For $\omega=\frac{1}{\sqrt{h+1}}$ and $c\left(y_{0}, y_{i}\right)=a>0$ for $i=1, \ldots, h$, the eigenvalues for $\mathcal{L}$ are,

$$
\mu_{1}=0, \mu_{j}=a, \mu_{h+1}=a(h+1),
$$

$j=2, \ldots, h$. The associated eigenfunctions $v_{k}$ are:

(i) When $\mu_{1}=0$, the eigenfunction is $v_{1}=\frac{1}{\sqrt{h+1}}$.

(ii) For $\mu_{j}=a, j=2, \ldots, h$, the eigenfunction is

$$
v_{j}\left(y_{k}\right)=\frac{1}{\sqrt{j(j-1)}}\left\{\begin{array}{cc}
1, & k=2, \ldots, j, \\
1-j, & k=j+1, \\
0, & \text { otherwise. }
\end{array}\right.
$$

(iii) For the eigenvalue $a(h+1)$ the eigenfunction is,

$$
v_{h+1}\left(y_{0}\right)=\sqrt{\frac{h}{h+1}}, \quad v_{h+1}\left(y_{k}\right)=-\frac{1}{\sqrt{(h+1) h}}
$$

for any $k=1, \ldots, h$.

In addition, to apply Theorem 5.6 we have to take into account the Green function of the Path. If $\lambda_{1}>0$, see [6], the Green function $G_{\lambda_{1}}^{V_{1}}$ is given by

$$
G_{\lambda_{1}}^{V_{1}}\left(x_{i}, x_{k}\right)=\frac{V_{i-1}(q) V_{m-k}(q)}{\lambda_{1} U_{m-1}(q)},
$$

where $q=1+\frac{\lambda_{1}}{2 c}$ and $G_{\lambda_{1}}^{V_{1}}\left(x_{i}, x_{k}\right)=G_{\lambda_{1}}^{V_{1}}\left(x_{k}, x_{i}\right)$ for any $1 \leq i \leq k \leq m$.

When $\lambda_{1}=0$, the expression for the Green function of the path with constant conductance is

$$
G^{V_{1}}\left(x_{i}, x_{k}\right)=\frac{3 i(i-1)+3(m-k)(m+1-k)+1-m^{2}}{6 m c},
$$

and $G^{V_{1}}\left(x_{i}, x_{k}\right)=G^{V_{1}}\left(x_{k}, x_{i}\right)$ for any $1 \leq i \leq k \leq m$.

So, in this context it would be possible to get an explicit expression of $G_{q}$ but it would include a lot of cases, according with vertices $y_{i}$, and it would have an awful appearance.

\section{Acknowledgment}

This work has been supported by the Spanish Research Council, Spain, under projects MTM2014-60450-R and MTM2017-85996-R. 


\section{References}

[1] C. Araúz, The Kirchhoff index of some composite networks, Discrete Appl. Math. 160 (2012) 1429-1440.

[2] C. Araúz, Á. Carmona, A.M. Encinas, Dirichlet-to-Robin maps on finite networks, Appl. Anal. Discrete Math. 9 (2015) 85-102.

[3] E. Bendito, A. Carmona, A.M. Encinas, Solving boundary value problems on networks using equilibrium measures, J. Funct. Anal. 171 (2000) 155-176.

[4] E. Bendito, A. Carmona, A.M. Encinas, Potential Theory for Schrödinger operators on finite networks, Rev. Mat. Iberoamericana 21 (2005) 771-818.

[5] E. Bendito, A. Carmona, A.M. Encinas, The Kirchhoff index of join networks, Discrete Appl. Math. 160 (2012) $24-37$.

[6] E. Bendito, A.M. Encinas, A. Carmona, Eigenvalues, eigenfunctions and Green's functions on a path via Chebyshev polynomials, Appl. Anal. Discrete Math. 3 (2009) 282-302.

[7] T. Biyikoğlu, J. Leydold, P.F. Stadler, Laplacian Eigenvectors of Graphs, in: LNM 1915, Springer, Berlin, 2007.

[8] A. Carmona, A.M. Encinas, M. Mitjana, Discrete elliptic operators and their Green operators, Linear Algebra Appl. 442 (2014) $115-134$.

[9] F. Chung, Spectral Graph Theory, in: CBMS Regional Conference Series in Mathematics, vol. 92, Amer. Math. Soc., Providence, RI, 1997.

[10] F. Chung, R.P. Langlands, A combinatorial Laplacian with vertex weights, J. Combin. Theory Ser. A 75 (1996) $316-327$.

[11] F. Chung, S.-T. Yau, Discrete Green's functions, J. Combin. Theory Ser. A 91 (1-2) (2000) 191-214.

[12] J.B. Conway, Functions of one Complex Variable, second ed., Springer-Verlag, New York, 1978.

[13] R.B. Ellis, Chip-firing Games with Dirichlet Eigenvalues and Discrete Green's Functions (Ph. D. Thesis), University of California at San Diego, 2002.

[14] R.B. Ellis, Discrete Green's functions on products of regular graphs. arXiv:math/0309080v2, 2003.

[15] A.C. Gilbert, J.G. Hoskins, J.C. Schotland, Diffuse scattering on graphs, Linear Algebra Appl. 496 (2016) 1-35.

[16] H. Zhang, Y. Yang, C. Li, Kirchhoff index of composite graphs, Discrete Appl. Math 157 (2009) 2918-2927. 